\title{
Inhibitory effect of dexamethasone on Lewis mice lung cancer cells
}

\author{
Y. Geng ${ }^{1 *}$, J. Wang ${ }^{1 *}$, H. Jing ${ }^{2}$, H.W. Wang ${ }^{1}$ and Y.X. Bao ${ }^{1}$ \\ ${ }^{1}$ Department of Respiratory Medicine, \\ Second Affiliated Hospital of Harbin Medical University, Harbin, China \\ ${ }^{2}$ Department of Emergency, \\ Second Affiliated Hospital of Harbin Medical University, Harbin, China \\ *These authors contributed equally to this study. \\ Corresponding author: Y.X. Bao \\ E-mail: gengyingz@126.com
}

Genet. Mol. Res. 13 (3): 6827-6836 (2014)

Received June 24, 2013

Accepted November 12, 2013

Published August 29, 2014

DOI http://dx.doi.org/10.4238/2014.August.29.4

\begin{abstract}
Recent studies have found that glucocorticoids are closely associated with oncogenesis and the development of many types of tumors. The aim of this study was to observe the effect of dexamethasone on the growth and angiogenesis of transplanted Lewis lung carcinoma in mice. Lewis lung carcinoma cells were inoculated subcutaneously into the right axilla of C57BL/6 mice, and the mice were randomly divided into 3 groups: the control group, cisplatin group, and dexamethasone group. From day 7 after inoculation, all the mice were given different treatments for 10 days, and changes in xenograft tumor volumes were monitored. All mice were sacrificed on day 17, and the tumors were obtained and weighed and the tumor inhibitory rate was calculated. The expression levels of hypoxia inducible factor $1 \alpha(\mathrm{HIF}-1 \alpha)$ and vascular endothelial growth factor (VEGF), as well as the microvessel density (MVD) in the tumor mass, were measured by immunohistochemistry. Tumor growth was suppressed in the cisplatin group and dexamethasone group. The weights of tumors were markedly decreased in the cisplatin group and dexamethasone group compared
\end{abstract}


with the control group $(\mathrm{P}<0.05)$. The expression levels of HIF-1 $\alpha$ and VEGF and the MVD were significantly lower in the cisplatin group and dexamethasone group than in the control group $(\mathrm{P}<0.05)$. However, these levels were not significantly different between the cisplatin group and dexamethasone group $(\mathrm{P}>0.05)$. Dexamethasone can effectively inhibit the growth and angiogenesis of Lewis lung carcinoma by inhibiting the expression of HIF- $1 \alpha$ and VEGF.

Key words: Dexamethasone; Lung carcinoma; Angiogenesis; Cyclin D1; Proliferating cell nuclear antigen; HIF-1 $\alpha$

\section{INTRODUCTION}

Lung cancer has the highest incidence and mortality among all cancers in the world and poses a serious threat to human health. Research on the possible mechanisms and prevention of lung cancer development and metastasis are especially important for improving the treatment of this disease. Glucocorticoids are steroid hormones secreted by the adrenal zona and can regulate growth, development, and differentiation and stabilize the internal environment. They play a regulatory role in the growth and differentiation of almost all cells.

Dexamethasone is a synthetic glucocorticoid that is widely used in the clinical setting to improve the general condition of cancer patients and reduce adverse reactions to chemotherapy. Recent studies have found that glucocorticoids are closely related to the development of a variety of tumors. Dexamethasone can inhibit the expression of vascular endothelial growth factor (VEGF) mRNA in induced human vascular smooth muscle cells (Nauck et al., 1998) and rat glioma cells (Machein et al., 1999) in vitro. This shows that dexamethasone has a certain anti-tumor and anti-angiogenic effect. In addition, dexamethasone can inhibit proliferation and induce differentiation in many tissue-derived carcinoma cells such as gynecologic and digestive system tumor cells (Arafa et al., 2006; Yemelyanov et al., 2008; Melhem et al., 2009). However, little research has been done on the inhibitory effect of dexamethasone on lung carcinoma cells. The aim of this study was to investigate the effect of dexamethasone on tumor growth, angiogenesis, cell proliferation, and related factors in lung tumors by establishing a C57BL/6 mouse xenograft model with Lewis lung carcinoma cells. We believe this will expand current methods of lung cancer treatment and provide a new basis for hormone therapy for lung tumors.

\section{MATERIAL AND METHODS}

\section{Animals}

Thirty specific pathogen-free (SPF) C57BL/6 mice were provided by the Experimental Animal Center of the Third Affiliated Hospital of Harbin Medical University. The mice were $18 \pm 2 \mathrm{~g}$ each, female, and 6-8 weeks old. This study was carried out in strict accordance with the recommendations in the Guide for the Care and Use of Laboratory Animals of the National Institutes of Health. The animal use protocol was reviewed and approved by the Institutional Animal Care and Use Committee of Harbin Medical University. 


\section{Establishment of the Lewis lung carcinoma model}

We cultivated Lewis lung carcinoma cells (Cell Bank of Type Culture Collection of Chinese Academy of Sciences, Shanghai, China) in Dulbecco's modified Eagle's medium containing $10 \%$ fetal bovine serum, and placed them in a $37^{\circ} \mathrm{C}$ culture incubator with $5 \% \mathrm{CO}_{2}$. We collected the cell suspension that comprised logarithmic growth phase cells, adjusting the cell density to $1 \times 10^{7} / \mathrm{mL}$ with phosphate-buffered saline. We then established the animal model by inoculating the cells into the right axilla of the mice. Every mouse was inoculated a with $0.2-\mathrm{mL}$ suspension containing $2 \times 10^{6}$ cells, and then fed under an SPF environment. Tumor growth was closely observed.

\section{Animal groups and processing}

Thirty C57BL/6 mice were divided equally into 3 groups at random and administered with drugs on the 7th day after inoculation. The mice of the control group were intraperitoneally administered saline. The mice of the second group were intraperitoneally administered $2 \mathrm{mg} / \mathrm{kg}$ cisplatin (Zhunzi H20023461; Shandong Qilu Pharmaceutical Company) dissolved in $0.2 \mathrm{~mL}$ saline once daily on days 7,8 , and 9 , and $0.2 \mathrm{~mL}$ saline alone was administered on the other days. The mice of the third group were intraperitoneally administered $5 \mathrm{mg} / \mathrm{kg}$ dexamethasone (Zhunzi H37020289; Shandong Xinhua Pharmaceutical Co., Ltd) dissolved in 0.2 $\mathrm{mL}$ saline once each day. Each group received treatment for 10 continuous days. We observed the eating habits, defecation, mental state, and activities of the mice during the course of the experiment. The subcutaneous tumor nodules of each mouse were measured on days 7, 9, 11, 13,15 , and 17 after inoculation using vernier calipers (accuracy of $0.1 \mathrm{~mm}$ ). We then recorded the size of the tumor and calculated the volume as follows: $\mathrm{V}\left(\mathrm{mm}^{3}\right)=0.52 \mathrm{ab}^{2}(\mathrm{a}=$ longest diameter of the nodule; $b=$ shortest diameter). The average of each group was used to plot the tumor growth curve.

On day 17 after inoculation, all mice were sacrificed by cervical dislocation. We obtained and weighed the subcutaneous tumors to calculate the tumor inhibition rate. The tumors were fixed with $4 \%$ paraformaldehyde, paraffin-embedded, and sectioned. The tumor inhibition rate was calculated as follows: $=$ (average tumor weight of the treatment group/average tumor weight of the control group) $\mathrm{x} 100 \%$.

\section{Immunohistochemistry}

Antibodies against hypoxia inducible factor $1 \alpha$ (HIF-1 $\alpha$ ), VEGF, VIII-R Ag, cyclin D1, and proliferating cell nuclear antigen (PCNA) were used for immunohistochemical analyses of tumor sections. The HIF-1 $\alpha$, VEGF, and cyclin D1 monoclonal antibodies were provided by Santa Cruz Biotechnology., USA. Both the VIII-R Ag antibody and the PCNA monoclonal antibody were obtained from Boshide Biotechnology Co. Ltd, Wuhan, China. The diaminobenzidine color kit and the streptavidin-peroxidase immunohistochemistry kit were provided by Zhongshan Golden Bridge Biotechnology Co. Ltd., Beijing, China. The procedure was performed according to manufacturer protocols. A positive result was determined by the number of positive cells and the staining intensity. A reference scoring method used (Mamori et al., 2007). Each section was observed with 10 random high-power field measure- 
ments under a microscope (400X magnification). The results were ranked according to the staining intensity of positive cells and the percentage of positive cells. The criteria for scoring staining intensity were as follows: no staining $=0$, slight staining $=1$, moderate staining $=2$, and deep staining $=3$. The criteria for scoring the percentage of positive cells were as follows: no positive cells $=0,<25 \%$ positive cells $=1,25-50 \%$ positive cells $=2$, and $>50 \%$ positive cells $=3$. The 2 scores were then added. Microvessel density (MVD) count was based on the method of Weidner (Weidner, 1995). We first scanned the entire slice to detect an area of high vascular density under a microscope at $40 \mathrm{X}$ or $100 \mathrm{X}$ magnification and then counted the number of microvessels in 5 horizons at 400X magnification. MVD was defined as the average of these counts. The microvessel counting standard specified that as long as a single cluster of endothelial cells or a branch structure of vascular stem cells, which stained brownish yellow with the VIII-R Ag antibody, were separated from nearby tumor cells, microvascular tissue, or connective tissue, all were regarded as one blood vessel.

\section{Analysis of cell cycle changes}

Fresh tumor tissue was cut into pieces after being washed with phosphate-buffered saline. The tissue was then digested with $0.25 \%$ trypsin, filtered through a 200 -mesh nylon net into a single cell suspension, and analyzed by flow cytometry (FACSCalibur, Becton Dickinson Biosciences, CA, USA). The results were analyzed using the CellQuest software (Becton Dickinson).

\section{Statistical analysis}

We analyzed the data using the SPSS 13.0 statistical software (SPSS Inc., IL, USA). Data are reported as means \pm standard deviation (SD); 1-way analysis of variance was used to compare the homogeneity of multi-group variance. $Q$ tests were used to compare pairwise means. In the case of unequal variances, a non-parametric test was used. We used a nonparametric method to compare multiple groups of count data. We analyzed the relationship between HIF- $1 \alpha$ and VEGF expression and MVD using Spearman rank correlation. P $<0.05$ was considered to be statistically significant.

\section{RESULTS}

\section{Tumor formation}

Seven days after inoculation, the tumors of mice were palpable subcutaneous nodules. The rate of tumor formation was $100 \%$. Subcutaneous tumors increased in volume and presented locally nodular growth.

\section{Subcutaneously transplanted tumor growth}

Tumors in the control group grew significantly faster than those in the other groups. The growth curve showed significant increase, but tumor growth in the treated groups gradually slowed down. Both treatment groups experienced inhibition of tumor growth (Figure 1). After 17 days, the differences in average volume and weight of tumors between each group 
were statistically significant $(\mathrm{P}<0.01)$. The tumor inhibition rates of the cisplatin and dexamethasone groups were 56.56 and $48.36 \%$, respectively. Tumors in the cisplatin group grew slower than those in the dexamethasone group, but this difference was not statistically significant $(\mathrm{P}>0.05)$ (Table 1).

Table 1. Average size, weight, and tumor inhibition rate of the transplanted tumors in the 3 groups of mice.

\begin{tabular}{lcccc}
\hline Group & Cases $(\mathrm{N})$ & Average tumor size $\left(\mathrm{mm}^{3}\right)$ & Average tumor weight $(\mathrm{g})$ & Tumor inhibition rate \\
\hline Control group & 10 & $3602.65 \pm 2041.35$ & $2.44 \pm 0.89$ & - \\
Cisplatin group & 10 & $561.60 \pm 437.15^{*}$ & $1.06 \pm 0.64^{*}$ & $56.56 \%$ \\
Dexamethasone group & 10 & $821.65 \pm 248.42^{* *}$ & $1.26 \pm 0.48^{* \#}$ & $48.36 \%$ \\
F & - & 19.287 & 11.593 & - \\
$\mathrm{P}$ & - & $<0.01$ & $<0.01$ & - \\
\hline
\end{tabular}

$* \mathrm{P}<0.01$, compared with the control group; \#P $>0.05$, compared with the cisplatin group.

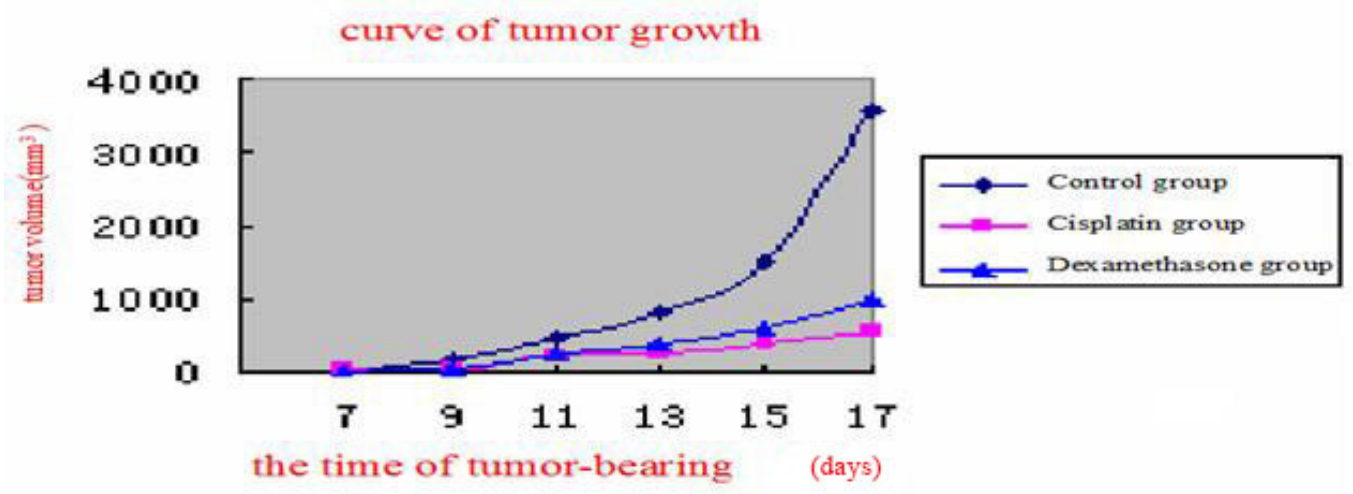

Figure 1. Tumor growth curve.

\section{HIF-1 $\alpha$ and VEGF expression and MVD}

Immunohistochemical analysis showed positive staining for HIF- $1 \alpha$ protein in the nucleus or cytoplasm of tumor cells. VEGF was mainly expressed in the cytoplasm of the tumor cells, and positive staining was characterized by intracytoplasmic, brown, and finely granular staining. Positive staining of vascular endothelial cells with VIII-R Ag antibody was brownishyellow (Figure 2A-I). Compared to the control and dexamethasone groups, expression scores of both HIF-1 $\alpha$ and VEGF as well as MVD counts were decreased in the cisplatin group; this difference was statistically significant on comparison with the control group $(\mathrm{P}<0.05)$ but was not significant on comparison with the dexamethasone group $(\mathrm{P}>0.05)$ (Table 2).

\section{Correlation analysis}

Spearman rank correlation analysis showed a significant positive correlation between VEGF and HIF-1 $\alpha$ expression and MVD in tumor tissue. The correlation coefficients were $\mathrm{r}=$ $0.881(\mathrm{P}<0.01)$ and $\mathrm{r}=0.725(\mathrm{P}<0.01)$, respectively. 
Table 2. Expression scores of HIF-1 $\alpha$ and VEGF and the microvessel density (MVD) count of tumor tissue in each group.

\begin{tabular}{lcccc}
\hline Group & Cases $(\mathrm{N})$ & HIF-1 $\alpha$ score & VEGF score & MVD count \\
\hline Control group & 10 & $4.3 \pm 1.25$ & $4.7 \pm 0.95$ & $39.66 \pm 4.50$ \\
Cisplatin group & 10 & $2.7 \pm 1.42^{*}$ & $2.6 \pm 1.43^{*}$ & $19.40 \pm 5.82^{*}$ \\
Dexamethasone group & 10 & $3.1 \pm 0.88^{\star \mathbf{\Delta}}$ & $3.4 \pm 1.07^{\mathbf{\Delta}}$ & $23.67 \pm 5.32^{* \mathbf{\Delta}}$ \\
F & & 4.788 & 8.220 & 12.456 \\
P & & 0.017 & 0.002 & 0.007 \\
\hline
\end{tabular}

Data are reported as means \pm SD. $* \mathrm{P}<0.01$, compared with the control group; $\# \mathrm{P}<0.05$, compared with the control group; $\boldsymbol{\Delta} \mathrm{P}>0.05$, compared with the cisplatin group.
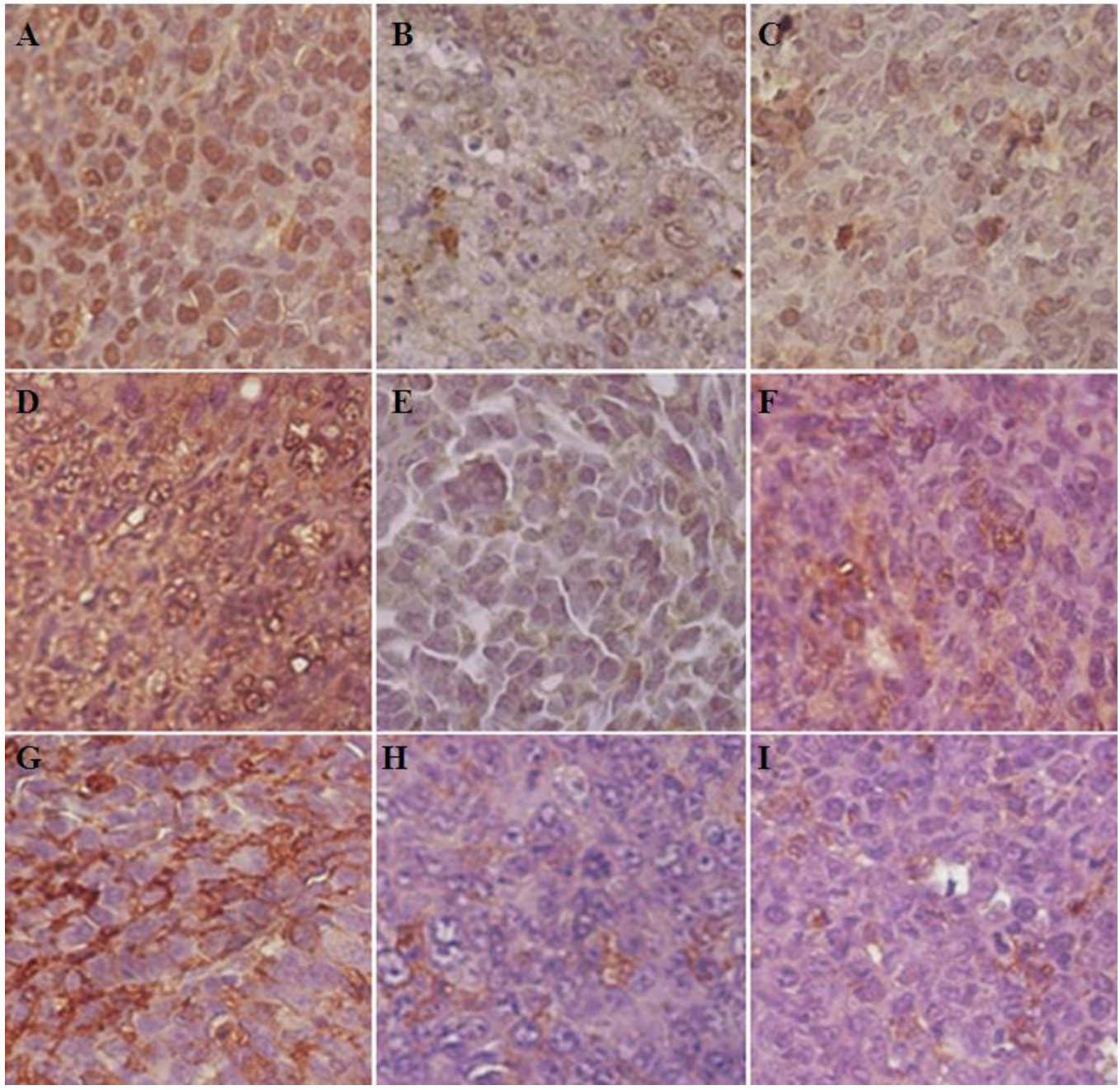

Figure 2. A. HIF-1 $\alpha$ staining in the control group, B. cisplatin group, C. dexamethasone group, D. VEGF staining in the control group, E. cisplatin group, F. dexamethasone group, G. MVD staining in the control group, H. cisplatin group, I. dexamethasone group. 


\section{Expression of cyclin D1 and PCNA protein}

The results of immunohistochemical analysis showed that expression levels of cyclin D1 and PCNA were significantly decreased in the dexamethasone and cisplatin groups when compared with expression in the control group, and this difference was statistically significant $(\mathrm{P}<0.05)$. However, comparison of the cisplatin and dexamethasone groups showed no significant difference $(\mathrm{P}>0.05)$ (Figure $3 \mathrm{~A}-\mathrm{F})$.
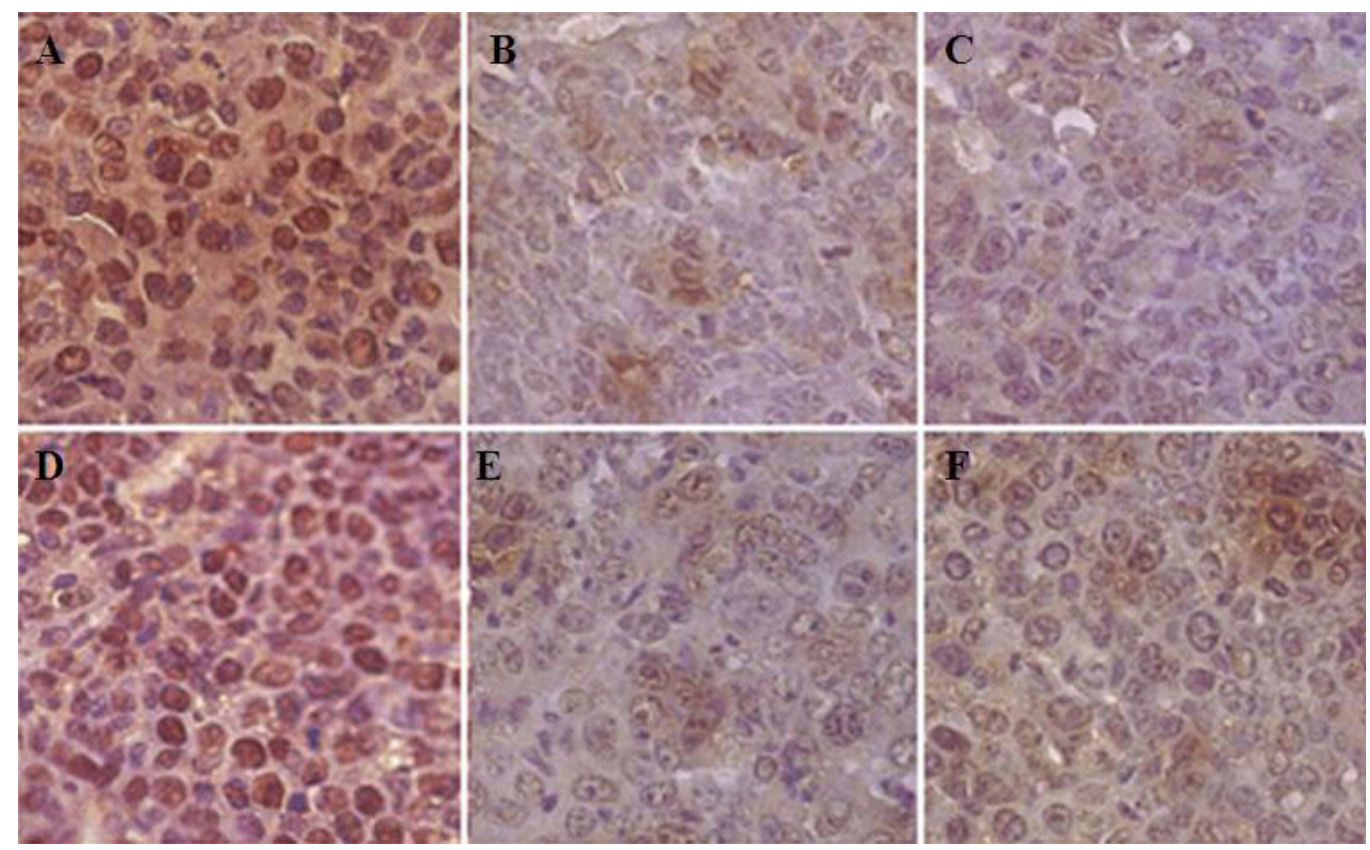

Figure 3. A. Expression of cyclin D1 in the control group, B. cisplatin group, C. dexamethasone group, D. expression of PCNA in the control group, E. cisplatin group, and $\mathbf{F}$. dexamethasone group..

\section{Changes in cell cycle}

The CellQuest software was used to analyze cell cycle phase distribution in each group. The percentage of cells in the G1 phase in the control group was $47.45 \% \pm 3.10 \%$, and the percentage of S-phase cells was $46.17 \% \pm 2.97 \%$. The percentage of cells in the G1 phase in the dexamethasone group was higher than that in the control group, at $63.76 \% \pm$ $1.85 \%$, and this difference was statistically significant $(\mathrm{P}<0.01)$. The percentage of cells in the $\mathrm{S}$ phase in the dexamethasone group was lower than that in the control group, at $32.13 \%$ $\pm 0.99 \%$, and this difference was also statistically significant $(\mathrm{P}<0.01)$. This result was similar to that seen in the cisplatin group (G1 phase: $68.41 \% \pm 2.53 \%$, S phase: $27.70 \%$ $\pm 2.39 \%$ ), but these percentages were not significantly different from those in the control group $(\mathrm{P}>0.05)$ (Figure 4A-C). 

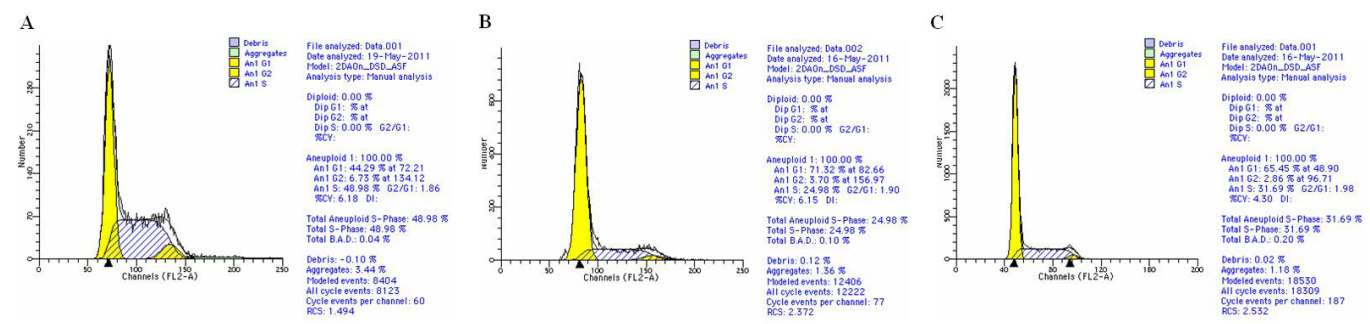

Figure 4. Cell cycle distribution detected by flow cytometry. A. Control group. B. Cisplatin group. C. Dexamethasone group.

\section{DISCUSSION}

Solid tumors grow vigorously, and the relative lack of blood supply can cause tissue hypoxia and a decrease in $\mathrm{pH}$. Therefore, tumor growth can promote the secretion of multiple cytokines. Tumor cells regulate the proliferation of mesenchymal cells through an intercellular signaling system, resulting in a special environment that is conducive to tumor cell proliferation and development. This is known as the tumor microenvironment, which mainly appears to be associated with extremely vigorous tumor angiogenesis (Fukumura and Jain, 2007). Therefore, theoretically, tumor treatments may include not only cytotoxic agents against tumor cells, but also agents targeted to inhibit the proliferation of or kill interstitial cells, such as vascular endothelial cells, which play a critical role in tumor growth. Since Folkman established the tumor angiogenesis theory, the importance of tumor angiogenesis to tumor growth is gradually being recognized (Folkman, 2007). This study confirmed that the inhibition of tumor angiogenesis can slow down tumor growth or even shrink tumor volume and reduce tumor burden (Folkman, 1971).

HIF- $1 \alpha$ is the most basic regulatory factor associated with cell responses under hypoxic conditions (Ferenci et al., 2010). HIF-1 $\alpha$ was first discovered by Semenza in 1992 when he studied hypoxia-inducible gene expression. HIF-1 $\alpha$ regulates the transcription and expression of numerous downstream genes, such as VEGF. It is the most important factor in hypoxia-induced transfer of gene transcription information (Wang and Semenza, 1993). VEGF is the most critical growth factor that stimulates tumor angiogenesis (Da et al., 2008). Its high expression links angiogenesis and tumor growth, and VEGF expression results in unlimited amplification of the tumor vasculature. In this condition, the growth of tumor cells cannot be controlled and metastasis of malignant cells is promoted. Thus, suppressing the action or expression of VEGF can inhibit tumor growth and metastasis. Studies showed that hypoxia results in high expression of VEGF in tumors and promotes angiogenesis in vivo through HIF-1 $\alpha$ (Liu et al., 1995; Kaelin, 2002). Tumor MVD is an important standard measure of angiogenesis. Tumors with a high degree of MVD appear to be strongly associated with malignancy, recurrence, and metastasis. Our results show that subcutaneous tumor growth was significantly inhibited in mice receiving dexamethasone. Not only was tumor growth significantly inhibited in the dexamethasone group, the MVD of tumor tissue in this group was also significantly lower than that in the control group. Expression levels of HIF-1 $\alpha$ and VEGF were lower than those in the control group, and the expression of both markers showed a significant positive correlation (Liao et al., 2001; Ushijima et al., 2001; Bremnes et al., 2006). By infer- 
ence, dexamethasone indirectly inhibits the generation of angiogenic factors such as VEGF by decreasing the expression of HIF-1 $\alpha$, thereby inhibiting tumor angiogenesis. This is an important role of dexamethasone in anti-angiogenic effects and suppression of tumor growth.

PCNA, a cofactor for the DNA polymerase $\delta$, is a type of nucleoprotein that is necessary for DNA synthesis within the nucleus. Proliferation signals from outside the cell are subject to the involvement of PCNA in order to facilitate DNA synthesis (Zhao et al., 2010). Thus, PCNA can mediate cell survival and proliferation. Studies have shown that the level of PCNA synthesis reflects the rate of cell proliferation and DNA synthesis during the entire cell cycle. Therefore, PCNA is a reliable marker of the proliferative phase during tumor growth, allowing for assessment of tumor cell proliferation (Yang and Zou, 2009). Cyclin D1 is a protein closely associated with cell proliferation, and it is mainly involved in the G1 phase of the cell cycle. It plays an extremely important role in the regulation of the cell cycle (Moghaddam et al., 2007). Our results suggested that dexamethasone significantly reduced PCNA and cyclin D1 expression, and with the reduction in cyclin D1 expression, mitotic complexes were not formed. Dexamethasone treatment arrested the cell cycle, preventing the transition from the G1 to $\mathrm{S}$ phase, which resulted in an accumulation of cells in the G1 phase, thereby inhibiting tumor cell proliferation.

In summary, dexamethasone can inhibit the growth of Lewis lung carcinoma and can reduce the side effects of chemotherapy. Dexamethasone is an ideal drug that can serve as the basis for tumor hormone therapy.

\section{ACKNOWLEDGMENTS}

Research supported by the Heilongjiang Provincial Education Department Project.

\section{REFERENCES}

Arafa HM, Abdel-Hamid MA, El-Khouly AA, Elmazar MM, et al. (2006). Enhancement by dexamethasone of the therapeutic benefits of cisplatin via regulation of tumor angiogenesis and cell cycle kinetics in a murine tumor paradigm. Toxicology 222: 103-113.

Bremnes RM, Camps C and Sirera R (2006). Angiogenesis in non-small cell lung cancer: the prognostic impact of neoangiogenesis and the cytokines VEGF and bFGF in tumours and blood. Lung Cancer 51: 143-158.

Da MX, Wu XT, Wang J, Guo TK, et al. (2008). Expression of cyclooxygenase-2 and vascular endothelial growth factor-C correlates with lymphangiogenesis and lymphatic invasion in human gastric cancer. Arch. Med. Res. 39: 92-99.

Ferenci P, Fried M, Labrecque D, Bruix J, et al. (2010). Hepatocellular carcinoma (HCC): a global perspective. J. Clin. Gastroenterol. 44: 239-245.

Folkman J (1971). Tumor angiogenesis: therapeutic implications. N. Engl. J. Med. 285: 1182-1186.

Folkman J (2007). Angiogenesis: an organizing principle for drug discovery? Nat. Rev. Drug Discov. 6: 273-286.

Fukumura D and Jain RK (2007). Tumor microvasculature and microenvironment: targets for anti-angiogenesis and normalization. Microvasc. Res. 74: 72-84.

Kaelin WG Jr. (2002). How oxygen makes its presence felt. Genes Dev. 16: 1441-1445.

Liao M, Wang H, Lin Z, Feng J, et al. (2001). Vascular endothelial growth factor and other biological predictors related to the postoperative survival rate on non-small cell lung cancer. Lung Cancer 33: 125-132.

Liu Y, Cox SR, Morita T and Kourembanas S (1995). Hypoxia regulates vascular endothelial growth factor gene expression in endothelial cells. Identification of a 5' enhancer. Circ. Res. 77: 638-643.

Machein MR, Kullmer J, Ronicke V, Machein U, et al. (1999). Differential downregulation of vascular endothelial growth factor by dexamethasone in normoxic and hypoxic rat glioma cells. Neuropathol. Appl. Neurobiol. 25: 104-112.

Mamori S, Nagatsuma K, Matsuura T, Ohkawa K, et al. (2007). Useful detection of CD147 (EMMPRIN) for pathological diagnosis of early hepatocellular carcinoma in needle biopsy samples. World J. Gastroenterol. 13: 2913-2917. 
Melhem A, Yamada SD, Fleming GF, Delgado B, et al. (2009). Administration of glucocorticoids to ovarian cancer patients is associated with expression of the anti-apoptotic genes SGKI and MKP1/DUSP1 in ovarian tissues. Clin. Cancer Res. 15: 3196-3204.

Moghaddam SJ, Haghighi EN, Samiee S, Shahid N, et al. (2007). Immunohistochemical analysis of p53, cyclinD1, RB1, c-fos and N-ras gene expression in hepatocellular carcinoma in Iran. World J. Gastroenterol. 13: 588-593.

Nauck M, Karakiulakis G, Perruchoud AP, Papakonstantinou E, et al. (1998). Corticosteroids inhibit the expression of the vascular endothelial growth factor gene in human vascular smooth muscle cells. Eur. J. Pharmacol. 341: 309-315.

Ushijima C, Tsukamoto S, Yamazaki K, Yoshino I, et al. (2001). High vascularity in the peripheral region of non-small cell lung cancer tissue is associated with tumor progression. Lung Cancer 34: 233-241.

Wang GL and Semenza GL (1993). Characterization of hypoxia-inducible factor 1 and regulation of DNA binding activity by hypoxia. J. Biol. Chem. 268: 21513-21518.

Weidner N (1995). Intratumor microvessel density as a prognostic factor in cancer. Am. J. Pathol. 147: 9-19.

Yang XH and Zou L (2009). Dual functions of DNA replication forks in checkpoint signaling and PCNA ubiquitination. Cell Cycle 8: 191-194.

Yemelyanov A, Czwornog J, Gera L, Joshi S, et al. (2008). Novel steroid receptor phyto-modulator compound a inhibits growth and survival of prostate cancer cells. Cancer Res. 68: 4763-4773.

Zhao Y, Li XJ, Sui X, Tang XJ, et al. (2010). Expression and significance of PCNA and Caspase-3 in the tissue of lung cancer. Xi Bao Yu Fen Zi Mian Yi Xue Za Zhi 26: 154-156. 